\title{
Bioactivity Study of Thiophene and Pyrazole Containing Heterocycles
}

\author{
NITIN V. KALE', SUPRIYA P. SALVE', BHAUSAHEB K. KARALE', SADHANA D. \\ MHASKE $^{2}$, SUSHAMA B. DARE ${ }^{1}$, ANIL E. ATHARE ${ }^{1}$ and SUSHAMA J. TAKATE ${ }^{1 *}$
}

'Department of Chemistry, New Arts, Commerce and Science College, Ahmednagar, 414001, affiliated to SPPU, Pune, Maharashatra, India.

${ }^{2}$ Department of Chemistry, Dadapatil Rajale Arts, Science and Commerce College, Adinathnagar, 414505, Affiliated to SPPU, Pune, Maharashtra, India.

*Corresponding author E-mail: sjtakate26@gmail.com

http://dx.doi.org/10.13005/ojc/370417

(Received: July 17, 2021; Accepted: August 19, 2021)

\section{ABSTRACT}

Chalcones 3a-f were prepared by reacting thiophene containing pyrazolyl aldehyde (2) with different 2-hydroxy acetophenones 1a-f. The compounds 3a-f were transformed into different Pyrazolines 4a-f. The formation of chromene derivatives 5a-f occurred from the cyclization of $\mathbf{3 a - f}$, which were then transformed into pyrazole derivatives 6a-f. Newly synthesized compounds have promising antibacterial activity against $S$. typhii and $S$. aureus, while weak activity against $B$. subtilis and $E$. coli. Compounds $\mathbf{5 d}$ and $\mathbf{6} \mathbf{d}$ had significant antifungal action towards $A$. niger, while most of the compounds were moderately active towards $T$. viride. Some of the synthesized compounds showed promising a-amylase inhibitory activity at $1 \mathrm{mg} / \mathrm{mL}$ concentration.

Keywords: 2-Hydroxyacetophenone, Pyrazole, Chromone, Antimicrobial activity.

\section{INTRODUCTION}

In the field of medicinal chemistry, most drugs have different heterocyclic scaffolds that show potential biological activities. Microbes are responsible for different human diseases. As these microorganisms develop resistance towards many of the present drug molecules, there is a need for continuous research on developing new potential medicinal agents. The presence of oxygen, sulphur and nitrogen containing heterocyclic nucleus imparts very effective pharmacological properties to therapeutic agents. In these scaffolds, the presence of Fluorine increases bioactivity of molecules several times ${ }^{1,2}$.

Thiophene derivatives have varied therapeutic applications. Thiophene containing heterocyclic compounds have created interest among the researchers owing to their vast spectrum of biological functions including antimicrobial ${ }^{3,4}$, antiparasitic $^{5,6}$, antiviral ${ }^{7}$, anticancer ${ }^{8,9}$, enzyme inhibitors ${ }^{10}$, anti-inflammatory and analgesic ${ }^{11}$ properties. Some of the commercially available drugs that contain thiophene as an integral component are Suprophen and Tiaprofenic acid

This is an Open Access article licensed under a Creative Commons license: Attribution 4.0 International (CC- BY). Published by Oriental Scientific Publishing Company @ 2018 
as an anti-inflammatory, Rolaxifene and OSI-930 as anticancer, Methapyrilene as anti-histamine, Tienilic acid as an antihypertensive, Ticlopidine as antiplatelet, Olanzapine as antisychotic, Etizolam as anti-anxiety and Tigabine as anticonvulsant agents.

In recent years, pyrazole is the most studied heterocycle among the azole family due to its innumerable chemical, agrochemical and pharmacological ${ }^{12,13}$ properties. Pyrazole containing compounds are reported for anticancer ${ }^{14,15}$ antibacterial, antifungal ${ }^{16-18}$, antiviral ${ }^{19,20}$, antinflammatory ${ }^{18,21}$, anti FAAH (Fatty Acid Amide Hydrolase) ${ }^{22}$, anti-enzymatic(Anti-S IRT 1 and SIRT 2) ${ }^{23}$, analgesic ${ }^{24}$, 5a-Reductase inhibitor ${ }^{25}$, antioxidant ${ }^{26,27}$, and insecticidal ${ }^{28}$. The Pyrazole scaffold has fascinating medicinal potential and is found as a pharmacophoric group of the drug molecules available on the market such as anti-inflammatory agents Meprizole, Celecoxib and Lonazolac, Rimonabant acts as cannabinoid receptor and used to treat obesity, Difenamizole functions as an analgetic, Fomepizole inhibits alcohol dehydrogenase, Fezolamine acts as antidepressant, CDPPB functions as anti-psychotic and sildenafil inhibits phosphodiesterase (Figure 1).

Chalcones have attracted much attention from medicinal chemists, not only as synthon for biosynthetic perspectives but also as bioactive moiety ${ }^{29-32}$. Several heterocyclic rings can be obtained from chalcones through ring closure reactions. Chalcone shows diversified medicinal and biological activities such as antimalarial ${ }^{33,34}$, anticancer ${ }^{35,36}$, anti-inflammatory ${ }^{37,38}$, antitubercular $^{39,40}$, Antioxidant ${ }^{41,42}$, anti-alzheimer ${ }^{43}$, antibacterial and antifungal ${ }^{44-46}$.

Chromones belong to the flavonoid family and are widely used in folk medicine because of their interesting biological activities ${ }^{47,48}$. The large number of bioactive molecules have chromone moiety as an essential pharmacophore ${ }^{49,50}$. Many of the biologically active chromones show anti-bacterial and antifungal ${ }^{51,52}$, anti-inflammatory ${ }^{53,54}$, antioxidant $^{55,56}$, anticancer ${ }^{57-59}$, anti-HIV ${ }^{60}$, anti-obesity ${ }^{61}$, antiviral ${ }^{62}$, antidiabetic ${ }^{48}$, anticonvulsant ${ }^{63}$ and anti-tubercular activities ${ }^{64}$. In light of the significance of thiophene, pyrazole, chalcone and chromone in numerous areas, particularly in medicinal chemistry (biological activity), the present study focuses on synthesis of these scaffolds and their biological activities.

Inhibition of the digestive enzymes is one of the approaches to manage diabetis. These enzymes catalyse the hydrolysis of starch into smaller carbohydrates like glucose ${ }^{65}$. This is one of the important steps in maintaining blood sugar level. In diabetics, hypoglycaemia can be achieved by inhibiting the enzyme alpha-amylase and the inhibitor can prove a potential anti-diabetic agent.

Heterocycles like flavones, pyrazoles and thiophenes are reported for their a-amylase inhibitory activity ${ }^{66-69}$.

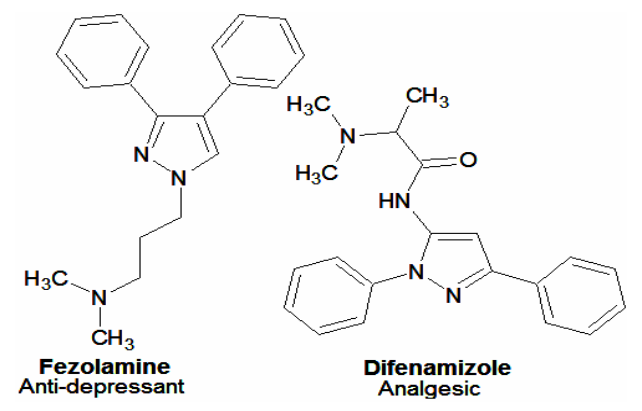

Fig. 1.

\section{RESULT AND DISCUSSION}

A well-known literature approach was used to synthesize various acetophenones (1a-f) and arylaldehyde (2) derivatives, as illustrated in Scheme-I and Scheme-II. The synthesis of chalcones, 3a-f was carried out by Claisen-Schmidt condensation by using 1a-f and 2. Different spectral methods have verified the formation of 3a-f. I.R. spectra of compound $\mathbf{3 d}$ shows band at $3439,1639 \mathrm{~cm}^{-1}$. HRMS shows a molecular ion peak at 459.0135 support $\mathbf{3 d}$ formation. ${ }^{1} \mathrm{HNMR}$ signal at 5.88 indicate olefinic proton and also the signal at $\delta 8.85$ shows proton of pyrazole ring. In ethanol, the molecule 3a-f interacts with hydrazine hydrate to produce bipyrazolyl phenols, 4a-f. This formation of pyrazolines 4a-f was confirmed by spectral technique. The I.R. Spectrum $3334 \mathrm{~cm}^{-1}$ and Molecular ion peak at 473.0410 in HRMS validated formation of $\mathbf{4 d}$. The most important confirmation of 4d formation is in ${ }^{1}$ HNMR spectra which shows two doublets at $\delta 3.17$ and $\delta 3.71$ confirm the presence of diastereotopic protons of methylene group of pyrazoline ring while the $\delta 5.09$ triplet of methine in pyrazoline ring. These signals strongly support the formation of 4a-f. Refluxing chalcone 3a-f in DMSO with a catalytic quantity of iodine yielded 2-substituted chromone 5a-f. The I.R. spectrum at $1653 \mathrm{~cm}^{-1}$ 
and the molecular ion peak at 456.9967 verify $\mathbf{5 d}$ ${ }^{1} \mathrm{HNMR}$ spectra validate chromone formation as there is absence of downfield signal above $\delta 10.0$ implies absence of $\mathrm{O}-\mathrm{H}$ and also singlet at $\delta 6.80$ is due to 3-position proton chromone. Chromones 5a-f when refluxed in ethanol and hydrazine hydrate were transformed into pyrazoles 6a-f. The I.R. Spectrum at $3404 \mathrm{~cm}^{-1}$ and In HRMS molecular ion peaks at 471.0243 supports $6 \mathrm{~d}$ formation. The most significant confirmation is the presence of a singlet at $\delta 12.70$ in ${ }^{1} \mathrm{HNMR}$ spectra of $\mathrm{O}-\mathrm{H}$ proton.

\section{Amylase Inhibitory Activity}

In a test tube $500 \mu \mathrm{L}$ of test sample, $500 \mu \mathrm{L}$ solution of a-amylase whose concentration is $0.5 \mathrm{mg} /$ $\mathrm{mL}$ and phosphate buffer of $0.2 \mathrm{mM}$ concentration were mixed and kept undisturbed $10 \mathrm{~min}$ at room temperature. Then the contents of the test tube were allowed to react $1 \%$ starch solution in phosphate buffer having $\mathrm{pH}$ 6.9. Then dinitrosalicylic acid was used to extinguish reaction. After incubation of $5 \mathrm{~min}$ test tubes were cooled and the contents were diluted with distilled water. The absorbance of resulting solutions was recorded at the wavelength $540 \mathrm{~nm}$. Then the results were compared with well-known inhibitor of the acarbose.

The results are recorded as \% inhibition of enzyme activity.

Table 1: Antimicrobial Activity (Zone of Inhibition at $1 \mathrm{mg} / \mathrm{mL}$ in $\mathbf{m m}$ )

\begin{tabular}{|c|c|c|c|c|c|c|}
\hline Compound & Bacillus subtilis & $\begin{array}{c}\text { Antibacterial Activity } \\
\text { Staphylococcus aureus }\end{array}$ & Salmonella typhii & Escherichia coli & $\begin{array}{l}\text { Antifungal Activity } \\
\text { Aspergillus niger }\end{array}$ & Trichoderma viride \\
\hline 2 & - & 11 & 13 & + & - & - \\
\hline $3 a$ & - & 13 & 12 & + & - & 13 \\
\hline $3 b$ & - & 15 & 13 & + & - & 14 \\
\hline $3 c$ & - & 14 & 18 & 10 & - & - \\
\hline $3 d$ & - & 10 & 11 & 10 & - & - \\
\hline $3 e$ & - & 11 & 12 & + & - & 12 \\
\hline $3 f$ & - & 12 & 12 & 10 & - & - \\
\hline $4 a$ & - & 13 & 13 & + & - & 11 \\
\hline $4 b$ & - & 12 & 15 & + & - & 14 \\
\hline $4 c$ & - & 11 & 12 & 10 & - & 13 \\
\hline $4 d$ & - & 12 & 13 & + & - & - \\
\hline $4 \mathrm{e}$ & - & 13 & 12 & + & - & 12 \\
\hline $4 f$ & - & 12 & 13 & + & - & - \\
\hline $5 a$ & - & 15 & 17 & 10 & - & 15 \\
\hline $5 b$ & - & 13 & 18 & + & - & 21 \\
\hline $5 c$ & - & 14 & 16 & + & - & - \\
\hline $5 d$ & - & 13 & 16 & + & 21 & 20 \\
\hline $5 e$ & - & 13 & 16 & 10 & - & - \\
\hline $5 f$ & - & 14 & 15 & 10 & - & - \\
\hline $6 a$ & - & 15 & 13 & 11 & - & - \\
\hline $6 \mathrm{~b}$ & - & 12 & 12 & 11 & - & - \\
\hline $6 c$ & - & 12 & 13 & 11 & - & - \\
\hline $6 d$ & - & 13 & 13 & 11 & 22 & 21 \\
\hline $6 e$ & - & 13 & 13 & 11 & - & 17 \\
\hline $6 f$ & - & 11 & - & - & - & - \\
\hline Ciprofloxacin & 28 & 23 & 40 & 26 & - & - \\
\hline Fluconazole & - & - & - & - & 28 & 29 \\
\hline
\end{tabular}

$\%$ Inhibition $=($ Ac-As $) / A c \times 100$

Where AC - Absorbance for Control and AS Absorbance for Sample

Table II: Amylase inhibitory Activity: (Concentration $1 \mathrm{mg} / \mathrm{mL}$ )

\begin{tabular}{cc}
\hline Compound & \% Inhibition \\
\hline $6 \mathrm{a}$ & 34 \\
$6 \mathrm{~b}$ & 31 \\
$6 \mathrm{e}$ & 09 \\
$6 \mathrm{f}$ & 10 \\
Acarbose & 45 \\
\hline
\end{tabular}

Microbial analysis

In vitro tests were performed on the compounds 2, 3a-f, 4a-f, 5a-f, and 6a-j against four bacterial and two fungal strains (Table 1). The agar well diffusion technique was utilized in this experiment. Ciprofloxacin and fluconazole were utilized as antibacterial and antifungal reference drugs respectively, while DMSO was used as a negative control. All compounds are inactive towards Gram-positive bacteria Bacillus subtilis and have modest action against Escherichia coli and Staphylococcus aureus. Compound $\mathbf{3 c}, \mathbf{5 a}$ and $\mathbf{5 b}$ showed good activity against Salmonella typhii while all other compounds have a moderate level of action. Compounds $\mathbf{5 d}$ and $\mathbf{6 d}$ were shown to have promising action against Aspergillus niger, while others had modest activity against Trichoderma viride. The results were averaged over three experimental sets and reported as zone of inhibition in millimeters. 
<smiles>[R]c1cc(O)c([R])c([R])c1[R]</smiles>

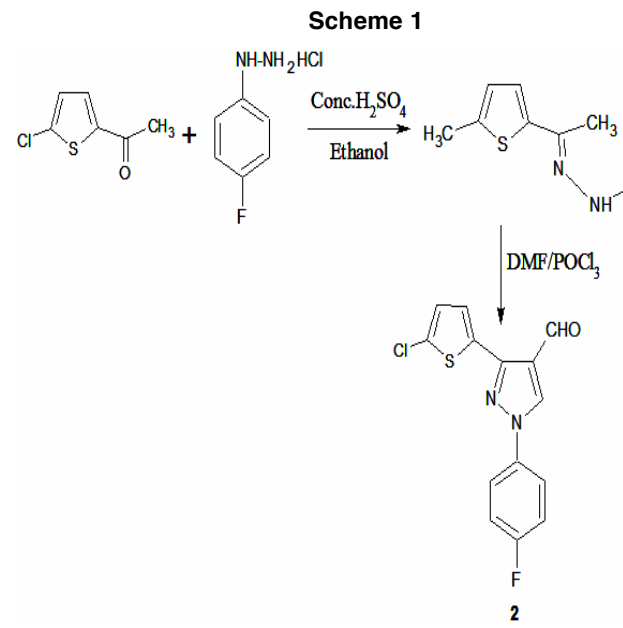

Scheme 2

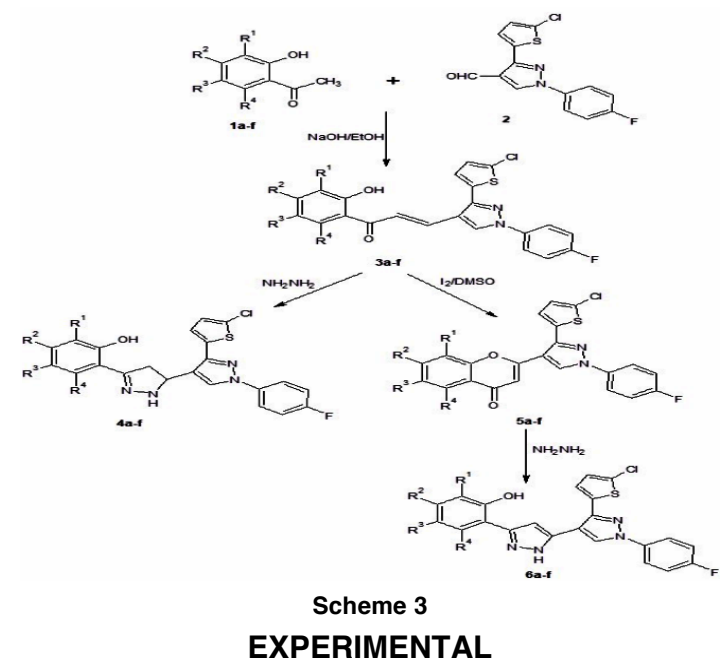

Open capillary technique was used for melting points which are uncorrected. IR Affinity-I Fourier transform infrared spectrophotometer (Shimadzu), Bruker Avance II $500 \mathrm{MHz}$ spectrophotometer and Waters SYNAPT G2 HDMS were used to record the IR, ${ }^{1} \mathrm{H}$ NMR and Mass spectra. Samples for NMR were prepared in DMSO- $d_{6}$ and TMS was internal reference. Absorption frequencies in terms of chemical shift were expressed in $\delta \mathrm{ppm}$. Mass spectra were recorded on.
Table 2: Physical data for synthesized compound

\begin{tabular}{ccccccc}
\hline Compound & $\mathrm{R} 1$ & $\mathrm{R} 2$ & $\mathrm{R} 3$ & $\mathrm{R} 4$ & m. p. $\left({ }^{\circ} \mathrm{C}\right)$ & Yield (\%) \\
\hline $3 \mathrm{a}$ & $\mathrm{H}$ & $\mathrm{H}$ & $\mathrm{H}$ & $\mathrm{H}$ & 170 & 65 \\
$3 \mathrm{~b}$ & $\mathrm{H}$ & $\mathrm{CH}_{3}$ & $\mathrm{H}$ & $\mathrm{H}$ & $172-174$ & 61 \\
$3 \mathrm{c}$ & $\mathrm{H}$ & $\mathrm{CH}_{3}$ & $\mathrm{Cl}$ & $\mathrm{CH}_{3}$ & $220-222$ & 63 \\
$3 \mathrm{~d}$ & $\mathrm{H}$ & $\mathrm{H}$ & $\mathrm{Cl}$ & $\mathrm{H}$ & $262-264$ & 66 \\
$3 \mathrm{e}$ & $\mathrm{Cl}$ & $\mathrm{H}$ & $\mathrm{Cl}$ & $\mathrm{H}$ & $280-282$ & 68 \\
$3 \mathrm{f}$ & $\mathrm{H}$ & $\mathrm{H}$ & $\mathrm{CH}_{3}$ & $\mathrm{H}$ & $262-264$ & 58 \\
$4 \mathrm{a}$ & $\mathrm{H}$ & $\mathrm{H}$ & $\mathrm{H}$ & $\mathrm{H}$ & $176-178$ & 84 \\
$4 \mathrm{~b}$ & $\mathrm{H}$ & $\mathrm{CH}_{3}$ & $\mathrm{H}$ & $\mathrm{H}$ & $188-190$ & 78 \\
$4 \mathrm{c}$ & $\mathrm{H}$ & $\mathrm{CH}_{3}$ & $\mathrm{Cl}$ & $\mathrm{CH}_{3}$ & $242-244$ & 86 \\
$4 \mathrm{~d}$ & $\mathrm{H}$ & $\mathrm{H}$ & $\mathrm{Cl}$ & $\mathrm{H}$ & $252-254$ & 82 \\
$4 \mathrm{e}$ & $\mathrm{Cl}$ & $\mathrm{H}$ & $\mathrm{Cl}$ & $\mathrm{H}$ & $294-296$ & 84 \\
$4 \mathrm{f}$ & $\mathrm{H}$ & $\mathrm{H}$ & $\mathrm{CH}_{3}$ & $\mathrm{H}$ & $264-266$ & 80 \\
$5 \mathrm{a}$ & $\mathrm{H}$ & $\mathrm{H}$ & $\mathrm{H}$ & $\mathrm{H}$ & $206-208$ & 76 \\
$5 \mathrm{~b}$ & $\mathrm{H}$ & $\mathrm{CH}_{3}$ & $\mathrm{H}$ & $\mathrm{H}$ & $218-220$ & 78 \\
$5 \mathrm{c}$ & $\mathrm{H}$ & $\mathrm{CH}_{3}$ & $\mathrm{Cl}$ & $\mathrm{CH}_{3}$ & $222-224$ & 80 \\
$5 \mathrm{~d}$ & $\mathrm{H}$ & $\mathrm{H}$ & $\mathrm{Cl}$ & $\mathrm{H}$ & $232-234$ & 82 \\
$5 \mathrm{e}$ & $\mathrm{Cl}$ & $\mathrm{H}$ & $\mathrm{Cl}$ & $\mathrm{H}$ & $256-258$ & 78 \\
$5 \mathrm{f}$ & $\mathrm{H}$ & $\mathrm{H}$ & $\mathrm{CH}_{3}$ & $\mathrm{H}$ & $248-252$ & 80 \\
$6 \mathrm{a}$ & $\mathrm{H}$ & $\mathrm{H}$ & $\mathrm{H}$ & $\mathrm{H}$ & $196-198$ & 82 \\
$6 \mathrm{~b}$ & $\mathrm{H}$ & $\mathrm{CH}_{3}$ & $\mathrm{H}$ & $\mathrm{H}$ & $204-206$ & 78 \\
$6 \mathrm{c}$ & $\mathrm{H}$ & $\mathrm{CH}_{3}$ & $\mathrm{Cl}$ & $\mathrm{CH}_{3}$ & $208-210$ & 72 \\
$6 \mathrm{~d}$ & $\mathrm{H}$ & $\mathrm{H}$ & $\mathrm{Cl}$ & $\mathrm{H}$ & $202-204$ & 76 \\
$6 \mathrm{e}$ & $\mathrm{Cl}$ & $\mathrm{H}$ & $\mathrm{Cl}$ & $\mathrm{H}$ & $218-220$ & 82 \\
$6 \mathrm{f}$ & $\mathrm{H}$ & $\mathrm{H}$ & $\mathrm{CH}_{3}$ & $\mathrm{H}$ & $212-214$ & 74 \\
\hline
\end{tabular}

(2E)-3-[3-(5-Chlorothiophen-2-yl)-1-(4-fluorophenyl)1H-pyrazol-4-yl]-1-(2-hydroxyphenyl)prop-2-en-1one, 3a

In $25 \mathrm{~mL}$ of ethanol and $12 \mathrm{~mL}$ of 30 percent $\mathrm{NaOH}$ solution, 2-hydroxyacetophenone 1a (0.015 mol) and 1,3-disubstituted-pyrazole-4-carbaldehyde $2(0.015 \mathrm{~mol})$ were dissolved and stirred at ambient temperature for 40-48 $\mathrm{h}$ with TLC monitoring. The contents were transferred to a beaker containing crushed ice. Then it was acidified using dil. acetic acid and the product was filtered and purified using alcohol to get $\mathbf{3 a}$. The compounds $\mathbf{3 b - 3} \mathbf{b}$ were prepared using the same procedure.

3a

Yellow Solid, IR (KBr): 3124, 3066, 2926, 1685, 1639, 1514, 831, $748 \mathrm{~cm}^{-1}$; HRMS: m/z $424.8751(\mathrm{M}+) ;{ }^{1} \mathrm{HNMR}: \delta 11.8(1 \mathrm{H}, \mathrm{s}), 8.89(1 \mathrm{H}, \mathrm{s})$, $7.95(2 \mathrm{H}, \mathrm{dd}, \mathrm{J}=9.12,4.12 \mathrm{~Hz}), 7.81(1 \mathrm{H}, \mathrm{dd}, \mathrm{J}=8.88$, $2.82 \mathrm{~Hz}), 7.71(1 \mathrm{H}, \mathrm{ddd}, \mathrm{J}=8.92,8.96,2.84 \mathrm{~Hz}), 7.46$ $(2 \mathrm{H}, \mathrm{t}, \mathrm{J}=9.12 \mathrm{~Hz}), 7.37(1 \mathrm{H}, \mathrm{d}), 7.28(1 \mathrm{H}, \mathrm{ddd}, \mathrm{J}=8.96$, $8.88,2.90 \mathrm{~Hz}), 7.21-7.24^{`}\left(3 \mathrm{H},{ }^{\prime} \mathrm{m}\right), 5.92(2 \mathrm{H}, \mathrm{q})$.

\section{3b}

Yellow Solid, IR(KBr): 3398, 3132, 3080, 1639, 1573, 1514, 833, $800 \mathrm{~cm}-1$; HRMS: m/z 438.9017(M+); ${ }^{1} \mathrm{HNMR}: \delta 10.2(1 \mathrm{H}, \mathrm{s}), 8.81(1 \mathrm{H}, \mathrm{s})$, 
$7.87(2 \mathrm{H}, \mathrm{dd}, \mathrm{J}=8.88,3.88 \mathrm{~Hz}), 7.71(1 \mathrm{H}, \mathrm{dd}, \mathrm{J}=8.64$, $2.58 \mathrm{~Hz}), 7.38(2 \mathrm{H}, \mathrm{t}, \mathrm{J}=8.88 \mathrm{~Hz}), 7.12-7.16(2 \mathrm{H}, \mathrm{m}), 7.09$ $(1 \mathrm{H}, \mathrm{dd}, \mathrm{J}=8.64,2.56 \mathrm{~Hz}), 5.84(2 \mathrm{H}, \mathrm{q}), 2.34(3 \mathrm{H}, \mathrm{s})$.

\section{$3 c$}

Yellow Solid, IR(KBr): 3352, 3124, 2924, 1672, 1639, 1585, 1514, 831, $752 \mathrm{~cm}^{-1}$; HRMS: $\mathrm{m} / \mathrm{z}$ 487.3733(M+); ${ }^{1} \mathrm{HNMR}: \delta 12.4(1 \mathrm{H}, \mathrm{s}), 8.93$ $(1 \mathrm{H}, \mathrm{s}), 7.99(2 \mathrm{H}, \mathrm{dd}, \mathrm{J}=9.14,4.14 \mathrm{~Hz}), 7.50(2 \mathrm{H}, \mathrm{t}$, $\mathrm{J}=9.14 \mathrm{~Hz}), 7.41(1 \mathrm{H}, \mathrm{d}), 7.24-7.28(2 \mathrm{H}, \mathrm{m}), \mathrm{i} 5.96 \mathrm{i}$ $(2 \mathrm{H}, \mathrm{iq}), \mathrm{i} 2.56 \mathrm{i}(3 \mathrm{H}, \mathrm{is}), \mathrm{i} 2.48$ (3H,is).

3d

Yellow Solid, IR(KBr): 3446, 3132, 3070, 1639, 1573, 1514, 833, $800 \mathrm{~cm}^{-1}$; HRMS: $\mathrm{m} / \mathrm{z}$ $459.0135(\mathrm{M}+) ;{ }^{1} \mathrm{HNMR}$ : $\delta 11.3(1 \mathrm{H}, \mathrm{s}), 8.85(1 \mathrm{H}, \mathrm{s})$, $7.91(2 \mathrm{H}, \mathrm{dd}, \mathrm{J}=9.4 \mathrm{~Hz}), 7.79(1 \mathrm{H}, \mathrm{d}, \mathrm{J}=2.7 \mathrm{~Hz}), 7.69$ $(1 \mathrm{H}, \mathrm{dd}, \mathrm{J}=8.8,2.72 \mathrm{~Hz}), 7.42(2 \mathrm{H}, \mathrm{t}, \mathrm{J}=9 \mathrm{~Hz}), 7.33$ $(1 \mathrm{H}, \mathrm{d}), 7.17-7.20(2 \mathrm{H}, \mathrm{m}), 5.88(2 \mathrm{H}, \mathrm{AB}-\mathrm{Quartet})$.

$3 e$

Yellow Solid, IR(KBr): 3446, 3130, 3070, 1643, 1570, 1508, 835, $808 \mathrm{~cm}^{-1}$; HRMS: $\mathrm{m} / \mathrm{z}$ 473.7652(M+); ${ }^{1} \mathrm{HNMR}$ : $\delta 10.8(1 \mathrm{H}, \mathrm{s}), 8.79(1 \mathrm{H}$, s), $7.85(2 \mathrm{H}, \mathrm{dd}, J=8.86,3.86 \mathrm{~Hz}), 7.71(1 \mathrm{H}, \mathrm{d}$, $J=2.56 \mathrm{~Hz}), 7.61(1 \mathrm{H}, \mathrm{d}, J=2.58 \mathrm{~Hz}), 7.36(2 \mathrm{H}, \mathrm{t}$, $J=8.86 \mathrm{~Hz}), 7.27(1 \mathrm{H}, \mathrm{d}), 7.11(1 \mathrm{H}, \mathrm{d}), 5.82(2 \mathrm{H}, \mathrm{q})$.

3f

Yellow Solid, IR(KBr): 3446, 3134, 1683, 1637, 1560, 1514, 831, $804 \mathrm{~cm}^{-1}$; HRMS: $\mathrm{m} / \mathrm{z}$ 438.9017(M+); ${ }^{1} \mathrm{HNMR}$ : $\delta 11.3(1 \mathrm{H}, \mathrm{s}), 8.91(1 \mathrm{H}$, s), $7.97(2 \mathrm{H}, \mathrm{dd}, J=9.06,4.06 \mathrm{~Hz}), 7.83(1 \mathrm{H}, \mathrm{d}$, $J=2.76 \mathrm{~Hz}), 7.73(1 \mathrm{H}, \mathrm{dd}, J=8.86,2.78 \mathrm{~Hz}), 7.49$ $(2 \mathrm{H}, \mathrm{t}, \mathrm{J}=9.06 \mathrm{~Hz}), 7.39(1 \mathrm{H}, \mathrm{d}), 7.22-7.26 \mathrm{i}(2 \mathrm{H}, \mathrm{im})$, i5.94i $(2 \mathrm{H}, \mathrm{iq})$, i2.32i(3H,is).

2-[(3S)-3'-(5-Chlorothiophen-2-yl)-1'-(4fluorophenyl)-3,4-dihydro-1'H,2H-3,4'-bipyrazol5-yl]phenol, 4a

A mixture of $2 \mathrm{~mL}$ hydrazine hydrate, $15 \mathrm{~mL}$ ethanol and substituted pyrazolyl chalcone $3 a(0.0015 \mathrm{~mol})$ was taken in R.B. flask and refluxed for 4 hours. Then by adding $2 \mathrm{~mL}$ of glacial acetic acid, heated for further 4 hours. Once the reaction was finished the contents were taken into crushed ice. The resulting product had been filtered. On crystallization from ethanol, pure compound $\mathbf{4 a}$ was obtained. The compound $\mathbf{4 b - 4 f}$ were prepared using the same procedure.
$4 a$

Yellow Solid, IR(KBr): 3309, 3084, 1593, 1514, 835, $802 \mathrm{~cm}^{-1}$; HRMS: m/z $438.9050(\mathrm{M}+)$; 'HNMR: $\delta i 12.26$ (s, 1H), 8.64i(s,i1H), i7.90-8.01 $(\mathrm{m}, \mathrm{i} 3 \mathrm{H}), 7.37-7.50(\mathrm{~m}, 4 \mathrm{H}), 7.32(1 \mathrm{H}$, ddd, $\mathrm{J}=8.84$, $8.82,2.68 \mathrm{~Hz}), 7.22(1 \mathrm{H}, \mathrm{d}, \mathrm{J}=8.92 \mathrm{~Hz}), 7.14(1 \mathrm{H}, \mathrm{ddd}$, $J=8.86,8.80,2.94 \mathrm{~Hz}), 6.97(1 \mathrm{H}, \mathrm{dd}, J=8.62,2.94 \mathrm{~Hz})$, $5.13(1 \mathrm{H}, \mathrm{t}, J=10.12 \mathrm{~Hz}), 3.75(1 \mathrm{H}, \mathrm{dd}, J=17.02$, $10.12 \mathrm{~Hz}), 3.21(1 \mathrm{H}, \mathrm{dd}, J=17.01 \mathrm{~Hz}, 10.12 \mathrm{~Hz})$.

$4 b$

Yellow Solid, IR(KBr): 3361, 3338, 3082, 1593, 1516, 829, $813 \mathrm{~cm}^{-1}$; HRMS: m/z $452.9316(\mathrm{M}+)$; 'HNMR: $\delta 11.64 \mathrm{i}(1 \mathrm{H}, \mathrm{is}), 8.56 \mathrm{i}(1 \mathrm{H}, \mathrm{is}), \mathrm{T} 7.84-7.93 \mathrm{i}(3 \mathrm{H}$, m), 7.29-7.44 (4H, m), 7.13 (1H, d, J=8.68Hz) $7.04(1 \mathrm{H}$, $\mathrm{dd}, J=8.62,2.52 \mathrm{~Hz}), 6.90(1 \mathrm{H}, \mathrm{d}, J=2.52 \mathrm{~Hz}), 5.05(1 \mathrm{H}$, t, $9.88 \mathrm{~Hz}), 3.67(1 \mathrm{H}, \mathrm{dd}, \mathrm{J}=17.04,9.88 \mathrm{~Hz}), 3.13(1 \mathrm{H}$, $\mathrm{dd}, \mathrm{J}=17.05,9.88 \mathrm{~Hz}), 2.28(3 \mathrm{H}, \mathrm{s})$.

$4 c$

Yellow Solid, IR(KBr): 3292, 3078, 1514, 835, $802 \mathrm{~cm}^{-1}$; iHRMS: m/z $501.4032(\mathrm{M}+)$; ${ }^{1} \mathrm{HNMR}$ : $\delta 10.74(1 \mathrm{H}, \mathrm{s}), 8.68(1 \mathrm{H}, \mathrm{s}), 7.94-8.05(3 \mathrm{H}, \mathrm{m})$, 7.41-7.54 $(3 \mathrm{H}, \mathrm{m}), 7.27(1 \mathrm{H}, \mathrm{d}, \mathrm{J}=8.94 \mathrm{~Hz}), 7.02(1 \mathrm{H}$ s), $5.17(1 \mathrm{H}, \mathrm{t}, \mathrm{J}=10.14 \mathrm{~Hz}), 3.79 \mathrm{i}(1 \mathrm{H}$,idd, $J=16.94$, $\mathrm{i} 10.14 \mathrm{~Hz}), 3.25(1 \mathrm{H}, \mathrm{dd}, J=16.94, \mathrm{i} 10.14 \mathrm{~Hz}), 2.40 \mathrm{i}$ $(3 \mathrm{H}$, is $)$, i2.30i( $3 \mathrm{H}, \mathrm{is})$.

\section{4d}

Yellow Solid, IR(KBr): 3334, 3084, 1514, 835, $817 \mathrm{~cm}^{-1}$; HRMS: $\mathrm{m} / \mathrm{z}$ 473.0410(M+); ${ }^{1} \mathrm{HNMR}$ : $\delta 11.68(1 \mathrm{H}, \mathrm{s}), 8.6(1 \mathrm{H}, \mathrm{s}), 7.86-7.97(3 \mathrm{H}, \mathrm{m}), 7.33-$ $7.46(4 \mathrm{H}, \mathrm{m}), 7.27(1 \mathrm{H}, \mathrm{dd}, \mathrm{J}=8.72,2.56 \mathrm{~Hz}), 7.21$ $(1 \mathrm{H}, \mathrm{d}, \mathrm{J}=8.8 \mathrm{~Hz}), 6.94(1 \mathrm{H}, \mathrm{d}, \mathrm{J}=8.5 \mathrm{~Hz}), 5.09(1 \mathrm{H}, \mathrm{t}$, $10 \mathrm{~Hz}), 3.71(1 \mathrm{H}, \mathrm{dd}, \mathrm{J}=17.01,10 \mathrm{~Hz}), 3.17(1 \mathrm{H}, \mathrm{dd}$, $J=17.01,10 \mathrm{~Hz}$ ).

$4 e$

Yellow Solid, IR(KBr): 3327, 3080, 1514, $835 \mathrm{~cm}^{-1}$; HRMS: m/z 507.7951 (M+); ${ }^{1}$ HNMR: $\delta$ $12.08(1 \mathrm{H}, \mathrm{s}), 8.54(1 \mathrm{H}, \mathrm{s}), 7.80-7.91(3 \mathrm{H}, \mathrm{m}), 7.27$ $7.40(4 \mathrm{H}, \mathrm{m}), 7.21(1 \mathrm{H}, \mathrm{d}, \mathrm{J}=2.42 \mathrm{~Hz}), 7.13(1 \mathrm{H}$, $\mathrm{d}, J=8.66 \mathrm{~Hz}), 5.03(1 \mathrm{H}, \mathrm{t}, 9.86 \mathrm{~Hz}), 3.65(1 \mathrm{H}, \mathrm{dd}$, $J=15.97,9.86 \mathrm{~Hz}), 3.11(1 \mathrm{H}, \mathrm{dd}, J=15.96,9.86 \mathrm{~Hz})$.

$4 f$

Yellow Solid, IR(KBr): 3277, 3093, 1514, 821, $804 \mathrm{~cm}^{-1}$; HRMS: $\mathrm{m} / \mathrm{z} 452.9316(\mathrm{M}+)$; ${ }^{1} \mathrm{HNMR}$ : ठ $10.14(1 \mathrm{H}, \mathrm{s}), 8.66(1 \mathrm{H}, \mathrm{s}), 7.92-8.04(3 \mathrm{H}, \mathrm{m})$, 7.39-7.52 $(4 \mathrm{H}, \mathrm{m}), 7.33(1 \mathrm{H}, \mathrm{dd}, \mathrm{J}=8.72,2.56 \mathrm{~Hz})$, $7.25(1 \mathrm{H}, \mathrm{d}, J=8.8 \mathrm{~Hz}), 7.00(1 \mathrm{H}, \mathrm{d}, J=8.5 \mathrm{~Hz}), 5.15$ 
(1H, t, J=10Hz), $3.77(1 \mathrm{H}, \mathrm{dd}, J=17.03,10 \mathrm{~Hz}), 3.23$ (1H, dd, J=17.03,10Hz), 2.30 (3H, s).

\section{2-[3-(5-Chlorothiophen-2-yl)-1-(4-fluorophenyl)- 1H-pyrazol-4-yl]-4H-chromen-4-one, 5a}

Substituted pyrazolyl propenone $\mathbf{3 a}(0.001$ mol) was refluxed in $12 \mathrm{~mL}$ of DMSO containing $0.2 \mathrm{~g}$ lodine at $135-145^{\circ} \mathrm{C}$ for $3-4 \mathrm{~h}$ and kept aside for 24 hours. Then the mixture was transferred to smashed ice and filtered solid was treated with 15\% sodium thiosulphate for removal of unreacted lodine. The compound $\mathbf{5 a}$ was purified by recrystallizing it from ethanol. The compound $\mathbf{5 b} \mathbf{b} \mathbf{5} \mathbf{f}$ were prepared using the same procedure.

\section{$5 a$}

Faint Solid, IR(KBr): 3124, 3059, 1653, 1516, $833 \mathrm{~cm}^{-1}$; HRMS: $\mathrm{m} / \mathrm{z} 422.8592(\mathrm{M}+)$; ${ }^{1} \mathrm{HNMR}$ : ס 8.00-8.05 $(4 \mathrm{H}, \mathrm{m}), 7.65(1 \mathrm{H}, \mathrm{dd}, \mathrm{J}=8.92,2.88 \mathrm{~Hz})$, $7.56(1 \mathrm{H}$, ddd, $\underline{J}=8.90,8.84,2.88 \mathrm{~Hz}), 7.49-7.53 \mathrm{i}$ $(4 \mathrm{H}, \mathrm{im}), \mathrm{i} .29 \mathrm{i}(1 \mathrm{H}, \mathrm{d}, \mathrm{i} J=3.92 \mathrm{~Hz}), 6.84(1 \mathrm{H}, \mathrm{is})$.

\section{$5 b$}

Yellowish Brown Solid, IR(KBr): 3122 , 3059, 1654, 1516, 833, $800 \mathrm{~cm}^{-1}$; HRMS: $\mathrm{m} / \mathrm{z}$ 436.8858(M+); ${ }^{1} \mathrm{HNMR}$ : $\delta$ 7.92-7.97 (4H, m), 7.57 $(1 \mathrm{H}, \mathrm{d}, J=2.42 \mathrm{~Hz}), 7.50(1 \mathrm{H}, \mathrm{dd}, J=8.56,2.42 \mathrm{~Hz})$, 7.41-7.44 $(3 \mathrm{H}, \mathrm{m}), 7.21(1 \mathrm{H}, \mathrm{d}, \mathrm{J}=3.68 \mathrm{~Hz}), 6.76(1 \mathrm{H}$, s), $2.38(3 \mathrm{H}, \mathrm{s})$.

\section{$5 c$}

Yellowish Brown Solid, IR(KBr): 3113 , 3057, 1645, 1514, 833, $792 \mathrm{~cm}^{-1}$; HRMS: $\mathrm{m} / \mathrm{z}$ 485.3575(M+); ${ }^{1} H N M R: ~ \delta ~ 8.04-8.09(3 \mathrm{H}, \mathrm{m}), 7.69$ $(1 \mathrm{H}, \mathrm{s}), 7.53-7.57(3 \mathrm{H}, \mathrm{m}), 7.33(1 \mathrm{H}, \mathrm{d}, \mathrm{J}=3.94 \mathrm{~Hz})$, $6.88(1 \mathrm{H}, \mathrm{s}), \mathrm{i} 2.60 \mathrm{i}(3 \mathrm{H}, \mathrm{is}), \mathrm{i} 2.54 \mathrm{i}(3 \mathrm{H}, \mathrm{s})$.

\section{5d}

Faint Yellow Solid, IR(KBr): 3140, 3066, 1653, 1541, 1517, 835, $796 \mathrm{~cm}^{-1}$; HRMS: $\mathrm{m} / \mathrm{z}$ 456.9967 (M+); ${ }^{1} \mathrm{HNMR}$ : $\delta$ 7.96-8.01 (4H, m), 7.61 $(1 \mathrm{H}, \mathrm{d}, \mathrm{J}=8.8 \mathrm{~Hz}), 7.45-7.49(4 \mathrm{H}, \mathrm{m}), 7.25(1 \mathrm{H}, \mathrm{d}$, $\mathrm{J}=3.8 \mathrm{~Hz}), 6.80(1 \mathrm{H}, \mathrm{s})$.

\section{$5 e$}

Yellow Solid, IR(KBr): 3124, 3074, 1654, 829, $779 \mathrm{~cm}^{-1}$; HRMS: $\mathrm{m} / \mathrm{z} 491.7494(\mathrm{M}+)$; ${ }^{1} \mathrm{HNMR}$ : ठ 8.02-8.07 $(4 \mathrm{H}, \mathrm{m}), 7.51-7.55(4 \mathrm{H}, \mathrm{m}), 7.31(1 \mathrm{H}$, d, $J=3.66 \mathrm{~Hz}), 6.86(1 \mathrm{H}, \mathrm{s})$.

\section{$5 f$}

Yellow Solid, IR(KBr): 3140, 3066, 1653,
1541, 835, $796 \mathrm{~cm}^{-1}$; HRMS: m/z $436.8858(\mathrm{M}+)$; ${ }^{1} \mathrm{HNMR}\left(\mathrm{DMSO}-d_{6}\right): \delta$ 7.90-7.96 $(4 \mathrm{H}, \mathrm{m}), 7.55$ $(1 \mathrm{H}, \mathrm{d}, \mathrm{J}=8.86 \mathrm{~Hz}), 7.39-7.43(4 \mathrm{H}, \mathrm{m}), 7.19(1 \mathrm{H}, \mathrm{d}$, $J=3.86 \mathrm{~Hz}), 6.74(1 \mathrm{H}, \mathrm{s}), 2.26(3 \mathrm{H}, \mathrm{s})$.

\section{2-[3'-(5-Chlorothiophen-2-yl)-1'-(4-fluorophenyl)- 1'H,2H-3,4'-bipyrazol-5-yl]phenol, $6 \mathrm{a}$}

In ethanol 2-substituted-chromen-4-one, 5a $(0.015 \mathrm{~mol})$ and hydrazine hydrate $(0.005 \mathrm{~mol})$ were dissolved and refluxed for 4 hours. TLC was used to monitor the reaction, and once it was finished, the reaction liquid stirred with cold water containing small amount of ice. After that, glacial acetic acid was used to neutralize the resulting mixture. Pure $6 \mathrm{a}$ was obtained by filtering the resultant product and recrystallizing it from ethanol. The compound $6 b-6 f$ were prepared using the same procedure.

\section{$6 a$}

Yellow Solid, IR(KBr): 3336, 3128, 3068, 1514, 835, $800 \mathrm{~cm}^{-1}$; HRMS: $\mathrm{m} / \mathrm{z} 436.8891$ (M+); ${ }^{1} \mathrm{HNMR}: \delta$ $13.12(1 \mathrm{H}, \mathrm{s}), 9.04(1 \mathrm{H}, \mathrm{s}), 8.14(1 \mathrm{H}, \mathrm{d}), 7.92-8.03(3 \mathrm{H}$, m), 7.37-7.49 $(5 \mathrm{H}, \mathrm{m}), 7.34(1 \mathrm{H}, \mathrm{ddd}, J=8.66,8.48$, 2.24Hz), $7.26(1 \mathrm{H}, d, J=9.20,2.24 \mathrm{~Hz}), 7.19(1 \mathrm{H}, \mathrm{d})$.

$6 b$

Yellow Solid, $\mathrm{R}(\mathrm{KBr})$ : 3421, 3186, 1514, 835, $698 \mathrm{~cm}^{-1}$; HRMS: m/z $450.9157(\mathrm{M}+)$; ' $\mathrm{HNMR}$ : $\delta 10.95(1 \mathrm{H}, \mathrm{s}), 8.96(1 \mathrm{H}, \mathrm{s}), 8.06(1 \mathrm{H}, \mathrm{d}), 7.84-7.95$ $(3 \mathrm{H}, \mathrm{m}), 17.47 \mathrm{i}(1 \mathrm{H}, \mathrm{ldd}, \mathrm{J}=8.33, \mathrm{i} 2.25 \mathrm{~Hz}), \mathrm{i} .29-7.39 \mathrm{i}$ $(4 \mathrm{H}, \mathrm{im}), 17.18 \mathrm{i}(1 \mathrm{H}, \mathrm{id}, \mathrm{iJ}=2.25 \mathrm{~Hz}), 17.13 \mathrm{l}(1 \mathrm{H}, \mathrm{Id})$, I2.19I(3H,Is).

$6 c$

Yellow Solid, IR(KBr): 3313, 3113, 1514 833, $802 \mathrm{~cm}^{-1}$; HRMS: $\mathrm{m} / \mathrm{z} 499.3873(\mathrm{M}+)$; ${ }^{1} \mathrm{HNMR}$ : ठ $11.37(1 \mathrm{H}, \mathrm{s}), 9.08(1 \mathrm{H}, \mathrm{s}), 8.18(1 \mathrm{H}, \mathrm{d}), 7.96-8.03$ $(2 \mathrm{H}, \mathrm{m}), 7.41-7.50(4 \mathrm{H}, \mathrm{m}), 7.30(1 \mathrm{H}, \mathrm{s}), 7.24(1 \mathrm{H}$, d), $2.55(3 \mathrm{H}, \mathrm{s}), 2.41(3 \mathrm{H}, \mathrm{s})$

\section{$6 d$}

Yellow Solid, IR(KBr): 3404, 3298, 3145, 1514, 833, $800 \mathrm{~cm}^{-1}$; HRMS: $\mathrm{m} / \mathrm{z} 471.0243(\mathrm{M}+)$ 1HNMR: $\delta 12.70(1 \mathrm{H}, \mathrm{s}), 9.0(1 \mathrm{H}, \mathrm{s}), 8.10(1 \mathrm{H}, \mathrm{d})$, 7.88-7.99 $(3 \mathrm{H}, \mathrm{m}), 7.33-7.45(5 \mathrm{H}, \mathrm{m}), 7.22(1 \mathrm{H}, \mathrm{d}$, $J=9.08 \mathrm{~Hz}), 7.17(1 \mathrm{H}, \mathrm{d})$.

$6 e$

Yellow Solid, IR(KBr): 3419, 3086, 1514, 835, $802 \mathrm{~cm}^{-1}$; HRMS: $\mathrm{m} / \mathrm{z}$ 505.7792(M+); ${ }^{1} \mathrm{HNMR}$ : $\delta 10.77(1 \mathrm{H}, \mathrm{s}), 9.06(1 \mathrm{H}, \mathrm{s}), 8.16(1 \mathrm{H}, \mathrm{d}), 7.94-8.06$ $(3 \mathrm{H}, \mathrm{m}), 7.39-7.51(5 \mathrm{H}, \mathrm{m}), 7.22(1 \mathrm{H}, \mathrm{d})$. 
$6 f$

Yellow Solid, IR(KBr): 3292, 3124, 1514, 835, $815 \mathrm{~cm}^{-1}$; HRMS: $\mathrm{m} / \mathrm{z} 450.9157(\mathrm{M}+)$; ${ }^{1} \mathrm{HNMR}$ : $\delta 12.15(1 \mathrm{H}, \mathrm{s}), 8.94(1 \mathrm{H}, \mathrm{s}), 8.04(1 \mathrm{H}, \mathrm{d}), 7.82-7.93$ $(3 \mathrm{H}, \mathrm{m}), 7.27-7.39(5 \mathrm{H}, \mathrm{m}), 7.17(1 \mathrm{H}, \mathrm{d}, \mathrm{J}=9.14 \mathrm{~Hz})$, $7.09(1 \mathrm{H}, \mathrm{d}), 2.12(3 \mathrm{H}, \mathrm{s})$.

\section{CONCLUSION}

Flourine and thiophene containing different pyrazolyl compounds were prepared in this present work and spectroscopic evidence strongly supports the suggested compounds. Compound $\mathbf{6 a}$ and $\mathbf{6 b}$ are promising alpha-amylase inhibitory activity in comparison with reference compound Acarbose. These compounds can be considered as lead compounds as anti-diabetic agents. Results of the antimicrobial study show that all the synthesized compounds can be modified structurally to improve their antimicrobial profile.

\section{ACKNOWLEDGEMENT}

The authors are thankful to Management of parent institute, Hon. Principal of the college, DST, Government of India for their support. The authors express their gratitude towards the Director, SAIF, Punjab University, Chandigarh, for spectroscopic investigations.

\section{Conflict of interest}

There are no conflicts of interest declared by the authors.

\section{REFERENCES}

1. Gillis, E. P.; Eastman, K. J.; Hill M. D. Donnelly, D. J.; Meanwell, N. A.; J. Med. Chem., 2015, 58(21), 8315-59 doi:10.1021/ acs.jmedchem.7b01788.

2. Meanwell, N. A.; J. Med. Chem., 2018, 61(14), 5822-80, doi:10.1021/acs.jmedchem. 5 b00258.

3. Abdel-Rahman, S. A.; El-Gohary, N. S; ElBendary, E. R. ; El-Ashry, S. M.; Shaaban, M. I.; Eur. J. Med. Chem., 2017, 140, 200-11 http:// dx.doi.org/10.1016/j.ejmech.2017.08.066.

4. Ajdačić, V.; Senerovic, L.; Vranić, M.; Pekmezovic, M.; Arsic-Arsnijevic, V.; Veselinovic, A.; Bioorg. Med. Chem., 2016, 24(6), 1277-91 http://dx.doi.org/10.1016/j. bmc.2016.01.058.

5. Félix, M. B.; de Souza, E. R.; de Lima, M. do CA.; Frade, D.K.G; Serafim, V. de L.; Rodrigues, K. A. da F.; Bioorg. Med. Chem., 2016, 24(18), 3972-7 http://dx.doi. org/10.1016/j.bmc.2016.04.057.

6. Jacomini, A. P.; Silva, M. J. V.; Silva, R. G. M.; Gonçalves, D. S.; Volpato, H.; Basso, E. A.; Eur. J. Med. Chem., 2016, 124, 340-9.

7. Desantis, J.; Nannetti, G.; Massari, S.; Barreca, M.L.; Manfroni, G.; Cecchetti, V.; Eur. J. Med. Chem., 2017, 138, 128-39 http:// dx.doi.org/10.1016/j.ejmech.2017.06.015.

8. Othman, D. I. A, Selim, K. B; El-Sayed, M. A. A.; Tantawy, A. S.; Amen, Y.; Shimizu, K.; Bioorg. Med. Chem., 2019, 27(19), 115026 https://doi.org/10.1016/j.bmc.2019.07.042.

9. Perin, N.; Rep. V.; Sović, I.; Juričić. Š.; Selgrad, D.; Klobučar, M.; Eur. J. Med. Chem., 2020, 185, 111833. https://doi. org/10.1016/j.ejmech.2019.111833.

10. Mandawad, G. G.; Dawane, B. S.; Beedkar, S. D.; Khobragade, C.N.; Yemul, O. S.; Bioorg. Med. Chem., 2013, 21(1), 365-72 http:// dx.doi.org/10.1016/j.bmc.2012.09.060.

11. Molvi, K. I.; Vasu, K. K.; Yerande, S. G.; Sudarsanam, V.; Haque, N.; Eur. J. Med. Chem., 2007, 42(8), 1049-58.

12. Ansari, A.; Ali, A.; Asif, M.; Shamsuzzaman, New J. Chem., 2016, 41(1), 16-41 http:// dx.doi.org/10.1039/C6NJ03181A.

13. Aziz, H.; Zahoor, A. F.; Ahmad, S. J.; Chil. Chem. Soc., 2020, 65(1), 4746-53.

14. Huang, Y. Y.; Wang, L. Y.; Chang, C. H.; Kuo, Y. H.; Kaneko, K.; Takayama, H.; Tetrahedron, 2012, 68(47), 9658-64 http:// dx.doi.org/10.1016/j.tet.2012.09.054.

15. Bertuzzi, G.; Locatelli, E,; Colecchia, D.; Calandro, P.; Bonini, B. F.; Chandanshive, J. Z.; Eur. J. Med. Chem., 2016, 117, 1-7 http:// dx.doi.org/10.1016/j.ejmech.2016.04.006.

16. Nayak, N.; Ramprasad, J.; Dalimba, U.; J. Fluor. Chem., 2016, 183, 59-68 http://dx.doi. org/10.1016/j.jfluchem.2016.01.011.

17. Viveka, S.; DineshaShama, P.; Nagaraja, G. K.; Ballav, S.; Kerkar, S.; Eur. J. Med. Chem., 2015, 101, 442-51 http://dx.doi.org/10.1016/j. ejmech.2015.07.002. 
18. Li, Y. R.; Li, C.; Liu, J. C.; Guo, M.; Zhang, T. Y.; Sun, L. P.; Bioorg. Med. Chem. Lett., 2015, 25(22), 5052-7.

19. Jia, H.; Bai. F.; Liu. N.; Liang, X.; Zhan, P.; Ma, C.; Eur. J. Med. Chem., 2016, 123, 202-10 http://dx.doi.org/10.1016/j.ejmech. 2016.07.048.

20. Fioravanti, R.; Desideri, N.; Biava, M.; Droghini, P.; Atzori, E. M.; lbba, C.; Bioorg. Med. Chem. Lett., 2015, 25(11), 2401-4 http://dx.doi.org/10.1016/j.bmcl.2015.04.006.

21. Khloya, P.; Kumar, S.; Kaushik, P.; Surain, P.; Kaushik, D.; Sharma, P. K.; Bioorg. Med. Chem. Lett., 2015, 25(6), 1177-81 http:// dx.doi.org/10.1016/j.bmcl.2015.02.004.

22. Aghazadeh, T. M.; Baraldi, P. G.; Ruggiero, E.; Saponaro, G.; Baraldi, S.; Romagnoli, R.; Eur. J. Med. Chem., 2015, 97, 289-305 http:// dx.doi.org/10.1016/j.ejmech.2015.04.064.

23. Therrien, E.; Larouche, G.; Nguyen, N.; Rahil, J.; Lemieux, A. M.; Li, Z.; Bioorg. Med. Chem. Lett., 2015, 25(12), 2514-8 http://dx.doi. org/10.1016/j.bmcl.2015.04.068.

24. Abd-EI, Gawad, N. M.; Hassan G. S.; Georgey, H. H.; Med. Chem. Re., 2012, 21(7) 983-94

25. Banday, A. H.; Shameem, S. A.; Jeelani, S. S.; Steroids., 2014, 13-9, http://dx.doi. org/10.1016/j.steroids.2014.09.004.

26. Kenchappa, R.; Bodke, Y. D.; Chandrashekar, A.; ArunaSindhe, M.; Peethambar, S. K.; Arab. J. Chem., 2017, 10, S3895-906 http:// dx.doi.org/10.1016/j.arabjc.2014.05.029.

27. Ambethkar, S.; Padmini, V.; Bhuvanesh, N.; J. Adv. Res., 2014, 6(6), 975-85, http://dx.doi. org/10.1016/j.jare.2014.11.011.

28. Liu, X. H.; Zhao, W.; Shen, Z. H.; Xing, J. H.; Yuan, J.; Yang, G.; Bioorg. Med. Chem. Lett., 2016, 26(15), 3626-8 http://dx.doi. org/10.1016/j.bmcl.2016.06.004.

29. Rani, A.; Anand, A.; Kumar, K.; Kumar, V.; Expert. Opin. Drug Discov., 2019, 14(3), 249-88 https://doi.org/10.1080/17460441.2 019.1573812 .

30. Zhuang, C.; Zhang, W.; Sheng, C,; Zhang, W.; Xing, C.; Miao, Z.; Chem. Rev., 2017, 117(12), 7762-810.

31. Gaonkar, S. L.; Vignesh, U. N.; Res. Chem. Intermed., 2017, 43(11), 6043-77.

32. Nowakowska, Z.; Eur. J. Med. Chem., 2007 42(2), 125-37.
33. Kumari, A.; Karnatak, M.; Singh, D.; Shankar, R.; Jat, J. L.; Sharma, S.; Eur. J. Med. Chem., 2019, 163, 804-29 https://doi.org/10.1016/j. ejmech.2018.12.007.

34. Kalaria, P. N.; Karad, S. C.; Raval, D. K.; Eur. J. Med. Chem., 2018, 158, 917-36 https://doi. org/10.1016/j.ejmech.2018.08.040.

35. Pontes, O.; Costa, M.; Santos, F.; SampaioMarques, B.; Dias, T.; Ludovico, P.; Eur. J. Med. Chem., 2018, 157, 101-14 https://doi. org/10.1016/j.ejmech.2018.07.058.

36. Karthikeyan, C.; NarayanaMoorthy, N. S. H.; Ramasamy, S.; Vanam, U.; Manivannan, E.; Karunagaran, D.; Recent. Pat. Anticancer Drug. Discov., 2014, 10(1), 97-115.

37. Bhale, P. S.; Chavan, H. V.; Dongare, S. B.; Shringare, S. N.; Mule, Y. B.; Nagane, S.; Bioorg. Med. Chem. Lett., 2017, 27(7), 1502-7 http://dx.doi.org/10.1016/j.bmcl.2017.02.052.

38. Li, J.; Li, D.; Xu, Y.; Guo, Z.; Liu, X.; Yang, H.; Bioorg. Med. Chem. Lett., 2017, 27(3), 602-606, 2018, 28(23-24), 3822, https://doi. org/10.1016/j.bmcl.2018.10.027.

39. Gomes, M. N.; Braga, R. C.; Grzelak, E. M.; Neves, B. J.; Muratov, E.; Ma, R.; Eur. J. Med. Chem., 2017, 137, 126-38 http://dx.doi. org/10.1016/j.ejmech.2017.05.026.

40. Chetty, S.; Ramesh, M.; Singh-Pillay, A.; Soliman, ME. S.; Bioorg. Med. Chem. Lett., 2017, 27(3), 370-86 http://dx.doi. org/10.1016/j.bmcl.2016.11.084.

41. Niu, H.; Wang, W.; Li, J.; Lei, Y.; Zhao, Y.; Yang, W.; Eur. J. Med. Chem., 2017, 138, 212-20 http://dx.doi.org/10.1016/j. ejmech.2017.06.033.

42. Tajammal, A.; Batool, M.; Ramzan, A.; Samra, M. M.; Mahnoor, I.; Verpoort, F.; J. Mol. Struct., 2017, 1148, 512-20 http://dx.doi. org/10.1016/j.molstruc.2017.07.042.

43. Wang, L.; Wang, Y.; Tian, Y.; Shang, J.; Sun, X.; Chen, H.; Bioorg. Med. Chem., 2017, 25(1), 360-71 http://dx.doi.org/10.1016/j. bmc.2016.11.002.

44. Besharati S. T.; Keivanloo, A.; Kaboudin, B.; Yoshida, A.; Yokomatsu, T.; Tetrahedron., 2018, 74(19), 2350-8 https://doi.org/10. 1016/j.tet.2018.03.055.

45. Yadav, P.; Lal, K.; Kumar, L.; Kumar, A.; Kumar, A.; Paul, A. K.; Eur. J. Med. Chem., 2018, 155, 263-74 https://doi.org/10.1016/j. ejmech.2018.05.055. 
46. Lal, K.; Yadav, P.; Kumar, A.; Kumar, A.; Paul, A. K.; Bioorg. Chem., 2018, 77, 236-44 https://doi.org/10.1016/j.bioorg.2018.01.016.

47. Ribnicky, D. M.; Poulev, A.; Watford, M.; Cefalu, W. T.; Raskin, I.; Phytomedicine., 2006, 13(8), 550-7.

48. Nurul, I. M.; Jung, H. A.; Sohn, H. S.; Kim, H. M.; Choi, J. S.; Arch. Pharm. Res., 2013, 36(5), 542-52.

49. Nazhand, A.; Durazzo, A.; Lucarini, M.; Romano, R.; Mobilia, M. A.; Izzo, A. A.; Nat. Prod. Res., 2020, 34(1), 137-52 https://doi. org/10.1080/14786419.2019.1678618.

50. Gaspar, A.; Matos, M. J.; Garrido, J.; Uriarte, E.; Borges, F.; Chem. Rev., 2014, 114(9), 4960-92 http://www.ncbi.nlm.nih.gov/ pubmed/24555663.

51. Kumar, S.; Koh, J.; Int. J. Mol. Sci., 2012, 13(5), 6103-16.

52. He, J.; Li, Z. H.; Ai, H. L.; Feng, T.; Liu, J. K.; Nat. Prod. Res., 2019, 33(24), 3515-20 https:// doi.org/10.1080/14786419.2018.1486313.

53. Huo, H. X.; Gu, Y. F.; Zhu, Z. X.; Zhang, Y. F.; Chen, X. N.; Guan, P. W.; Phytochemistry., 2019, 158, 46-55 https://doi.org/10.1016/j. phytochem.2018.11.003.

54. Venkateswararao, E.; Manickam, M.; Boggu, P.; Kim, Y.; Jung, S. H.; Bioorg. Med. Chem., 2015, 23(10), 2498-504 http://dx.doi. org/10.1016/j.bmc.2015.03.045.

55. Demetgül, C.; Beyazit, N.; Carbohydr. Polym., 2018, 181, 812-7 https://doi.org/10.1016/j. carbpol.2017.11.074.

56. Reis, J.; Cagide, F.; Valencia, M. E.; Teixeira, J.; Bagetta, D.; Pérez, C.; Eur. J. Med. Chem., 2018, 158, 781-800 https://doi.org/10.1016/j. ejmech.2018.07.056.

57. Demir, S.; Özen, C.; Ceylan-Ünlüsoy, M.;
Öztürk, M.; Bozdağ-Dündar, O.; J. Heterocycl. Chem., 2019, 56(4), 1341-51

58. Duan, Y. di.; Jiang, Y. yan.; Guo, F. xia.; Chen, L. xiao.; Xu, L. lu.; Zhang, W.; Fitoterapia., 2019, 135, 114-29.

59. Zhao, L.; Yuan, X.; Wang, J.; Feng, Y.; Ji, F.; Li, Z.; Bioorg. Med. Chem., 2019, 27(5), 677-85 https://doi.org/10.1016/j.bmc.2019.01.027.

60. Ungwitayatorn, J.; Wiwat, C.; Samee, W.; Nunthanavanit, P.; Phosrithong, N.; J. Mol. Struct., 2011, 1001(1-3), 152-61 http:// dx.doi.org/10.1016/j.molstruc.2011.06.035.

61. Iyengar, R. R.; Lynch, J. K.; Mulhern, M. M.; Judd, A. S.; Freeman, J. C.; Gao, J.; Bioorg. Med. Chem. Lett., 2007, 17(4), 874-8.

62. Zhang, Y.; Zhong, H.; Lv, Z.; Zhang, M.; Zhang, T.; Eur. J. Med. Chem., 2013, 62, 158-67.

63. Abbott, B. M.; Thompson, P. E.; Bioorg. Med. Chem. Lett., 2006, 16(4), 969-73.

64. Mujahid, M.; Gonnade, R. G.; Yogeeswari, P.; Sriram, D.; Muthukrishnan, M.; Bioorg. Med. Chem. Lett., 2013, 23(5), 1416-9 http:// dx.doi.org/10.1016/j.bmcl.2012.12.073.

65. Dhital, S.; Warren, F. J.; Butterworth, P. J.; P. R. Ellis and M. J. Gidley., 2014, doi.org/10.1 080/10408398.2014.922043.

66. Liu, X.; Luo, F.; Li, P.; She, Y.; Gao, W.; Food Res. Inter., 2017 doi: 10.1016/j.foodres. 2017.03.023.

67. Ibrahim, S. R. M.; Mohamed, G. A.; Abdel-latif, M. M. M.; El-Messerrey, S. M.; Al Musayeib, N. M.; Shehata, I. A.; Starch., 2015, DOI 10.1002/star.201500068.

68. Bhosale, M. R.; Deshmukh, A. R.; Pal, S.; Srivastava, A. K.; Mane, R. A.; Bioorg. Med. Chem. Lett., 2015, 25, 2442-2446.

69. Kumar, P.; Duhan, M.; Kadyan, K.; Sindhu, J.; Kumar, S.; Sharma, H.; Med. Chem. Comm., 2017, DOI: 10.1039/C7MD00080D. 